\title{
SYMMETRY PRINCIPLES IN OLD AND NEW PHYSICS
}

\author{
BY EUGENE P. WIGNER
}

Introduction and summary. Symmetry and invariance considerations have long played important roles in physics. The 32 crystal classes-that is, groups of rotations in three-dimensional space all the elements of which are of the order $2,3,4$ or 6 -were determined 137 years ago, in the same year in which group theory was born. The determination of the 230 space groups, by Schönflies and by Fedorov (these are the discrete subgroups of the Euclidean group which contain three noncoplanar translations) was a masterpiece of analysis and so was the determination by Groth of the possible properties of crystals with the symmetries of these space-groups.

The groups of prime importance in classical physics were subgroups of the Euclidean group and the enumeration of these subgroups and the derivation of the properties which are invariant under them were the principal problems. The invariance groups of the relativity theories were, from the mathematical point of view, much more esoteric but their use by physicists did not contribute greatly to the mathematical theory of groups nor did it point to new interesting mathematical problems. When, however, the invariance arguments were applied to the present century's other great innovation of physical theory, to quantum theory, a score of new problems and several interesting mathematical theorems were uncovered. The basic reason is the difference in the characterization of states in quantum and in pre-quantum theories. In the latter, a state was characterized by the positions and the velocities of particles. These could be specified by points in three-dimensional space. Quantum theory, on the other hand, specifies the states by vectors in an abstract Hilbert space. Symmetry transformations in pre-quantum theories were rather obvious transformations of three-dimensional space; in quantum theory they became unitary transformations of Hilbert space. These form subgroups of all unitary transformations which are essentially homomorphic to the symmetry group in question, essentially homomorphic only because a unitary transformation in quantum mechanics is equivalent to any of its multiples by a numerical factor (of modulus 1). However, this essential homomorphy could be reduced, particularly

This is the text of the forty-first Josiah Willard Gibbs lecture delivered before the Annual Meeting of the Society in San Francisco on January 23, 1968; received by the editors April 25, 1968. 
as a result of Bargmann's investigations [12], in most cases to a true homomorphy to an extended group which is called, then, the quantum mechanical symmetry group. The quantum mechanical operations of the symmetry group break up the Hilbert space of all states into subspaces each of which is invariant under the operations in question. These operations form, then, within each invariant subspace, a representation of the quantum mechanical symmetry group by means of unitary transformations.

The physicists undertook a much more detailed investigation of the representations of the groups in question than the mathematicians had been interested in previously. In those cases in which the representations were known before in principle, they defined canonical forms of the irreducible representations and determined the invariant subspaces of the direct products (also called tensor products) of invariant subspaces. This led to the theories of the various coupling coefficients, such as three, six, and higher $j$-coefficients which are of interest from the point of view of pure mathematics also. For several noncompact Lie groups, the unitary representations of which were not known before, these were determined. In many cases, all of the not trivial ones were infinite dimensional. The determination of these for all locally compact groups became a field of mathematical interest.

The theory of group representations was, until a few years ago, at the center of the interest of the physicist investigating the consequences of invariance principles. The invariance group was, in most cases, either the quantum mechanical Poincaré group (LC2I), or a subgroup thereof. In later years, the interest shifted to groups which are only approximate symmetry groups and form extensions of the Poincaré group. Hence, the interest shifted back from the representations of definite groups to the determination of groups, in particular those which contain the Poincaré group as a subgroup. Many interesting results were obtained concerning the existence of such groups and also their representations.

The evolution of the physical sciences. Physics and the natural sciences have changed enormously during the past 100 or 150 years. The spirit has changed, the subject has changed, and the mode of operation has changed.

The change in spirit has been toward increasing sophistication. Whereas a hundred or so years ago, the laws of physics were formulated in terms of directly observable quantities, present day physics uses intricate mathematical constructs-indeed, its analysis of the concept of "directly observable quantities" led to the conclusion that, 
in the microscopic domain, there is no such thing. It is easy to forget that the first big step in the direction of mathematical sophistication, the introduction of phase space with its billions of dimensions, was due to Willard Gibbs [1]. We all are keenly aware of the other two most important steps: the establishments of the relativity and quantum theories. It is not devoid of the elements of irony that the last two steps were undertaken in order to eliminate not directly observable quantities from the physical theory. What was accomplished is to have eliminated quantities, the impossibility of the direct observation of which was then recognized. What was substituted eventually for these quantities, the state vector and the gravitational metric, are not directly observable either; they are vastly more sophisticated than the earlier concepts both mathematically and conceptually.

The subject of physics, and of the other natural sciences, has changed also. The change can be most easily specified in the case of physics: whereas until the turn of the century physics was concerned only with macroscopic objects, we now consider an article on macroscopic physics, such as the wonderful new theory of friction, that of Bowden and Tabor [2], to be esoteric.

Finally, the mode of operation has changed. We now have Alvin Weinberg's Big Science, with hundred of scientists streaming in to the Laboratories each morning to tear away the covers with which nature seeks to hide its secrets. This change has been discussed a greal deal and I do not want to add to the discussion now.

What has not changed is that, as in Galileo's time, and in Galileo's words [3], the laws of nature are spoken in the language of mathematics. What also has not changed-at least not in the last 150 years -is that the concepts of symmetry, of invariance, play a very large role and, it appears, an increasing role in physics.

The parts of mathematics which are of most use to physicists have, on the other hand, changed enormously. Even 50 years ago, the mathematics of the physicist consisted of ordinary differential equations, with a sprinkling of partial ones. The latter, and the theory of Hilbert space, assumed the dominant position about 35 years ago. Nowadays, however, we hear more about the theory of analytic functions, of one or more variables, of the theory of distributions and, last but not least, the theory of groups and their representations, than the theory of Hilbert space and principal axis transformations which, after all, still are believed to provide the language for expressing what we know about the laws of nature.

Crystal symmetry. Let us now turn to the first subject proper: 
the role of symmetry in very old physics. This was, essentially, confined to the discipline of crystallography. The reason for my discussing it is not that I have new mathematical results on the subject but that the history of crystallography illustrates the development of the same ideas, side by side, by mathematicians and by natural scientists, first knowing very little about each other, but interacting vigorously later. The natural scientist's role is, to a considerable extent, to furnish the original problems and some of their solutions. The mathematician not only gives a deeper understanding of the solution given by the natural scientist but also greatly generalizes the initial problem. In the early stages, the two disciplines do not know about each other's methods and conclusions; the interaction becomes intimate in the later stages.

My story will be, naturally, mostly confined to the natural scientist whose story may be novel to mathematicians.

It begins in 1830-just 138 years ago-when J. F. C. Hessel determined the 32 crystal classes [4]. These are the finite groups of rotations in three-space, proper and improper, which have only elements of the order 1, 2, 3, 4, and 6 . This was just two years before a famous duel took place in Paris and in the same year in which the name group was coined and its concept was precisely formulated.

One may wonder why Hessel confined his attention to groups with elements all of the order $1,2,3,4,6$. The reason was that he knew of a property of crystals, discovered by one of the founders of crystallography, the Abbé Haüy, about 50 years earlier [5]. This law refers to crystallographic planes. It stipulates that, using the three intersections of any three crystal planes as directions of the coordinate axes, the ratio of the intercepts of any crystal plane with respect to these axes, if measured in terms of the intercepts of any other crystal plane, are rational numbers. This so-called law of rational indices is incompatible with any rotational symmetry except rotations by 60 and 90 degrees, and multiples of these angles. This leads to Hessel's condition of all symmetry elements of the rotation group being of order $1,2,3,4$, or 6 .

Haüy's law of rational indices was an empirical law and, needless to say, it could not have been established were it not true that the rational numbers referred to therein are very simple. If the basic planes are properly chosen, both the numerators and the denominators of the rational numbers are, as a rule, below six, of ten they are below four. If one were to admit arbitrary rational numbers, the law could not have been verified experimentally. The law could not have been invented at all without the picture of the structure of the 
crystal, conceived almost 300 years ago (by the bishop, Steno [6]), as a regular, lattice-like arrangement of atoms as we now know it to be. This picture led Haüy to the law of rational indices which was then experimentally verified and this, in its turn, led to the restriction of the rotations, which can be crystal symmetries, to those used by Hessel. When the total symmetries of crystal lattices, that is symmetries including spatial displacements, were investigated just before the turn of the century, by Fedorov and by Schönflies [7], Hessel's groups appeared as the 32 different factor groups of all possible space groups, the displacements forming the normal subgroups. The space groups are discrete subgroups of the Euclidean group which contain three noncoplanar translations. There are 230 of them, as determined by Schönflies and by Fedorov-by means of group theoretical methods which became, by that time, familiar at least to some crystallographers. Hessel's restriction of the groups, to those with elements of orders $1,2,3,4$, and 6 , was much less arbitrary than might at first appear.

The rest of the story of the role of symmetry in very old physics is interesting only for us physicists. The consequences of the symmetry properties of crystals in terms of the macroscopic properties yielded a fascinating amount of information-information that was derived, principally by Groth [8], with a full understanding of the group property of symmetry operations though, as a rule, not in the language of group theory. It continues to be a pleasure for most of us older physicists to read about the properties of crystals, also to learn about the very few exceptions in which the symmetry, present in the overwhelming number of properties, is violated by a few. It is painful, on the other hand, to admit that the symmetry cannot be formulated in terms of our present, quantum mechanical theory, that it is surely only approximate. Approximate in the sense that it is valid if classical, that is nonquantum, theory is a valid approximation for the motion of the nuclei. It is painful also to admit that none of us has succeeded in finding the limits of the validity of the concept of crystal symmetry and to point to phenomena in which the approximate nature of the symmetry would manifest itself.

Before turning to more modern subjects, and in particular to quantum theory, it may be worth observing that even though the explicit role of symmetry in very old physics was largely confined to crystallography, the intuitive concept of symmetry probably played a great role in the thinking of the early great physicists. Thus, the force between two point-like bodies was assumed to be central, i.e., to have the direction of the line connecting the two bodies. This is the 
only direction compatible with rotational invariance. The invariance of the laws of physics with respect to translations - of both space and time-was an assumption which pervaded the thinking of natural scientists much before a sophisticated language for its formulation was invented. Some writings of Newton clearly show his awareness of the principle now called Galilean invariance.

At the opposite end of the period under review, Hamel, Klein, and Nöther gave highly elegant and sophisticated derivations of the conservation laws of physics on an invariant theoretic basis. These conservation laws were, of course, well known by that time on the basis of elementary derivations. For this reason, and because the application of the symmetry principle in question is a rather indirect one, and also because the subject has been discussed a great deal before by both mathematicians and physicists, it will not be further elaborated here.

Quantum mechanics. It must have been surprising that symmetry principles played an explicit and direct role only in a very restricted part of very old physics: in crystallography. Surely, the invariance of the equations of motion with respect to the whole Galilei or Poincaré groups [9], including as they do the whole Euclidean group, must have direct consequences for phenomena outside of crystallography. Let us see what those consequences are.

Let us consider a very simple system, a hydrogen atom with the electron moving in a Bohr orbit. What are the obvious and simpleminded consequences of the invariance of the equations of motion with respect to the Galilei or the Poincare groups? First, that the hydrogen atom could be anywhere else rather than where it actually is and that the electron could be further ahead or farther back on its orbit. Further, that the atom could be in uniform straightforward motion rather than at rest as I imagined it to be. Finally, that the orbit could be tilted in some way in space, rather than being horizontal. All this may be true, but none of this provides us with any of the properties of the orbit.

Let us consider the last conclusion. It is true, perhaps, that the hydrogen atom could be anywhere and could have any velocity. It is surely not true that its orbit could have any orientation in space. If it could, then even a hydrogen atom at rest at a given position could be in infinitely many entirely distinct states which is contrary to all intuition and all experience and also to the vanishing entropy of the internal motion. Everyone felt this, much before the advent of quantum mechanics.

How does quantum mechanics solve this contradiction? Once we 
know the state of motion of the hydrogen atom, we know its state completely. It then follows that the hydrogen atom (at least if we disregard its spin) is spherically symmetric. Are, however, all the states of the hydrogen atom spherically symmetric? Evidently not; a proton-electron combination must have not-spherically-symmetric states. Since, just as in classical theory, all such states can be subjected to a rotation, there are infinitely many states connected with most excited states of the hydrogen atom. However, and this is the crucial point, all these states can be written as linear superpositions of a finite number of states. The essential point here is that, in quantum mechanics, the physical state, the actual situation of a system, is characterized not by positions and velocities but by a vector in Hilbert space [10]. The equivalence of all directions does entail, in quantum as well as in classical theory, the existence of all states which are obtained from any given state by a rotation. However, unlike in classical theory, this does not conclude the story. In quantum theory, all the states obtained by the rotation of one can be written as linear combinations of certain basic states; in classical theory the linear superposition of states does not exist. The states in quantum theory are vectors in a linear space-which they are not in classical theory. We cannot add two states of a classical system to each other; we can do this for quantum states. Furthermore, the state vector which is the sum of two other state vectors is not really a new state; it is, with a probability $\frac{1}{2}$ the first state, with a probability $\frac{1}{2}$ the second. This is true at least if the state vectors of the latter states are orthogonal; if they are not orthogonal to begin with, they are not entirely different from each other either. It follows from this, in particular, that, when calculating the entropy, only the states with orthogonal state vectors must be counted.

Before formulating the mathematical problems to which this situation, the linear character of the state vectors, leads us, it may be well to complete the preceding picture in some regard. Thus far, we have considered only rotations of a definite state. How about imparting to them a certain velocity? Let us consider the hydrogen atom, first at rest, then moving with various velocities. It follows from the Galilei or the Poincare invariance that, if a system at rest is conceivable, the same system can be in uniform motion with any velocity, in any direction. We again have to postulate, as a result of the existence of a given state, an infinity of other states, just as in classical theory. Is it true again that, in quantum mechanics, this infinity of states, or rather their state vectors, can be represented as a linear combination of a smaller number of states? No, the answer is, in this case, in the 
negative; in fact, the state vectors are orthogonal for all pairs of states with different velocities. This is natural from the point of view of physics - one can experimentally distinguish states with different velocities though not with different orientations of the orbits-but it is surprising to find such a difference between the consequences of two types of invariance transformations: rotations in space and conferring a velocity.

Let us look, finally, at the last type of Galilei transformations: displacements in space and time. The situation with regard to these is very simple: if the velocity is specified, the state is invariant with respect to displacements. This is a consequence of Heisenberg's uncertainty relation. If one wishes to obtain a state localized at a given time near a point, one has to form a superposition of states with different velocities-states which we have just learned to be orthogonal to each other.

It may be good to repeat in precise mathematical terminology what was said before in the language of the physicist. The state of a system is given in classical mechanics by a point in phase space. This has six dimensions if the system consists of a single particle: the coordinates are the positional coordinates and the velocity components of the particle. The phase space has correspondingly more dimensions if the system consists of more particles [11]. The Galilei and Poincaré transformations are linear inhomogeneous transformations in phase space. In quantum mechanics, on the other hand, the state of any system is characterized by a vector in infinite dimensional Hilbert space. The invariance transformations are linear, in fact unitary, transformations in that space. Since the unitary transformation which corresponds to the product of two invariance transformations is, at least essentially, the product of the unitary transformations which correspond to the two factors of the product, these unitary transformations form, at least essentially, a unitary representation of the symmetry group. The symmetry group is the Galilei group in nonrelativistic theories; it is the Poincare group in relativistic theories, but it can be a subgroup of these if some outside influence decreases the total symmetry of space-time. Thus, for the motion of the electrons in a crystal, the symmetry group is one of the 230 space groups which were discussed before.

The great difference between classical and quantum transformations is, however, not the difference between a linear inhomogeneous transformation on the one hand, and a unitary one on the other. The great difference is, first, that in nonquantum theory the transformation is always the same, or, rather, depends only on the number 
of particles the positions and velocities of which we have to transform. In quantum theory, on the other hand, the symmetry transformations, the unitary representation of the symmetry group, are different for different systems; they determine many of the properties of the system. The difference is also that the addition of two states is meaningless in nonquantum theory but meaningful in quantum theory; the state space of quantum theory, its Hilbert space, is a truly linear space.

The first point goes far in explaining the physicist's interest in unitary representations. The unitary representations of the Galilei group are implicit already in Schrödinger's theory; they were shown to be implicit most clearly by Bargmann [12]. The unitary representations of the Poincaré group were determined in the late '30s; except for the trivial one, they were all shown to be infinite dimensional [13]. This is equivalent with the statement that no system can be relativistically invariant unless it can be in an infinity of orthogonal states. By calling attention to the properties of the unitary representations of noncompact Lie groups, the physicists have stimulated the mathematicians' interest in this subject. The mathematicians are now very much ahead of us in this field, and it is not easy to catch up with the results of Gelfand, Neumark, Harish-Chandra, and of many others [14].

The role of the group of rotations in three-space. Let us now return to a question which was alluded to before: the difference between the effect of rotations and that of imparting velocities, to a state. One can say that the total invariance group is composed of three types of elements: displacements, rotations, and the imparting of a velocity. If we consider minimal subspaces which are invariant under displacements, that is, the subspaces of a representation space which form the basis of an irreducible representation of the displacement subgroup, there is one such subspace which remains invariant under rotations. This does not surprise the physicist: the subspace in question is the one the state vectors of which describe the system at rest. In fact, in the relevant irreducible representations of the whole symmetry group, the effect of the rotations on this subspace is that of an irreducible representation of the group of rotations. The transformations which correspond to the imparting of a velocity, on the other hand, transform each minimal subspace which is invariant under displacements into a similar subspace which is, however, orthogonal to the subspace to the states of which a velocity was imparted. Thus, imparting of a velocity has an effect on these subspaces similar to that 
which any transformation has in classical theory: it produces an entirely new state. In a sense, therefore, the velocity imparting transformations are trivial, the transformations which correspond to rotations highly nontrivial: they form, for the subspace in question, an irreducible representation of the rotation group. It follows that the irreducible representations of the total symmetry group can be characterized by the behavior of the particular, preferred, minimal subspace under the influence of displacements and of rotations. This question was discussed in so much detail in order to give an explanation for the physicist's intense interest in the group of rotations in three-space, the explanation of this interest starting from the general Poincaré or Galilei invariance principle.

If we think of an irreducible representation of the rotation group, or any other group, and want a firm grasp on it, it is good to define a coordinate system in the representation space. The way this can be done most naturally is to specify a sequence of subgroups, $G, G_{n-1}$, $G_{n-2}, \cdots, G_{1}$ each being a maximal subgroup of the preceding one and $G_{1}$ being the group consisting of the unit element only. Let us assume then that the transformations of $G$ which correspond to elements of $G_{n-1}$ which form, therefore, a representation of this subgroup $G_{n-1}$, contain no irreducible representation of $G_{n-1}$ more than once. Let us further assume that the same is true of all irreducible representations of each $G_{k}$, if restricted to the subgroup $G_{k-1}$. Then, we can specify a direction in the representation space of $G$ uniquely by enumerating the irreducible representations of $G_{2}, G_{3}, \cdots, G_{n-1}$ in the representation spaces of which this direction is contained. We can then specify a unit vector in this direction; the unit vectors obtained in this way will be used as basis vectors in the representation space. They will be orthogonal to each other and will form a complete set of vectors in the space of the irreducible representation of $G$ from which we started.

An example may illustrate the situation. Let us choose for $G$ the symmetric group of all permutations of $n$ symbols, $S_{n}$. If we choose as the sequence of subgroups the groups $S_{n-1}, S_{n-2}, \cdots, S_{1}$ where $S_{n-k}$ is the symmetric group which leaves the last $k$ symbols unchanged, it follows from the classical theories of Young and Frobenius [15] that indeed no irreducible representation of $S_{n-k}$, if restricted to the subgroup $S_{n-k-1}$, will contain any representation of the latter more than once. Hence, one can specify, for instance, a vector in the space of the representation $3+2$ of $S_{5}$ by stipulating that it belong to the representation space of the representation $3+1$ of $S_{4}$, to the representation space $2+1$ of $S_{3}$, and to the representation 2 of $S_{2}$. In the 
case of the group of rotations in three-space, the sequence of subgroups contains, in addition to $O_{3}$, only $O_{2}$ (and $O_{1}$ ), $O_{2}$ being the group of rotations which leave the $z$ axis invariant, i.e., the rotations about the $z$ axis. This leads to the usual form of the irreducible representations of $O_{8}$.

Before leaving this subject, I would like to mention a new mathematical result related to the preceding consideration [16]. It is a necessary and sufficient condition that a subgroup have the property considered before: that the restriction of the representation to this subgroup contain no representation of the subgroup more than once, and that this be true for all irreducible representations of the original group. In order to formulate the condition, it is useful to introduce the concept of a subclass. This is the set of elements which can be transformed into each other by the elements of the subgroup. It is clear that the product of two subclasses consists of complete subclasses, that the ordinary classes of the group consist of one or more subclasses. It is not clear that the subclasses commute as do the ordinary classes. If they do, the subgroup has the property specified above and this is also a necessary condition therefor. It is easy to see that the subclasses will commute if they are self-inverse and it is easy to show that the subclasses of $S_{n}$ with respect to the subgroup $S_{n-1}$ are self-inverse. Hence, the result of the theories of Young and Frobenius, which was mentioned before, can be obtained in this way also.

The definite form of the irreducible representations of the three dimensional rotation group helps to solve some concrete problems. The squares of the matrix elements have a simple interpretation which is, though, difficult to verify experimentally: they give the probability that a particle with a definite component of its angular momentum in one direction have a given angular momentum component in another direction. Better known, and amply verified, are the Hönl-Kronig rules for the intensity ratios of the transitions between the sublevels into which two levels are split by a magnetic field [17]. The subgroup $O_{2}$ must be chosen in this case to be the rotations which leave the direction of the magnetic field unchanged.

Decomposition of the tensor products of representations. The definite form of the irreducible representations also helps in the solution of the problem to which we turn next: the reduction of the Kronecker product (also called inner tensor product) of representations into their irreducible components. It would be difficult to enumerate all the applications of this reduction. The physical basis 
of most of the applications is the rule for obtaining the state vector of the union of two systems: this is defined in the Hilbert space which is the direct product (also called Kronecker product) of the Hilbert spaces of the separate systems and the state vector is the Kronecker product of the state vectors of the individual systems [18]. Hence, if two systems are united, each being in a state which is one of the basis vectors of an irreducible representation - not necessarily the same irreducible representation-the state vector of the union of the two systems will be in the representation space of the Kronecker product of the two irreducible representations. A similar statement applies if three or more physical systems are united. The first question, from an invariant theoretic point of view, is then: how can the product space be decomposed into subspaces, each of which belongs to an irreducible representation, and what are these irreducible representations?

It may be useful to sketch the way in which an answer to these questions was found. The most important group to which the considerations can be and were applied is again $\mathrm{O}_{3}$. Somebody said that, unlike the mathematician, the physicist lives in three-dimensional space not only physically but also intellectually. $O_{3}$ is an ambivalent group, that is, all its classes are self-inverse. This renders all its characters real and this will be assumed in what follows. The complications which not-real characters entail are minor. The Poincaré group is ambivalent, but some of the groups to be considered later are not.

If the characters of the representations of which we wish to form the direct product are $\chi^{(a)}, \chi^{(b)}, \cdots$, the character of their direct product is

$$
\Xi(r)=\chi^{(a)}(r) \chi^{(b)}(r) \chi^{(o)}(r) \cdots
$$

and the number of times $\chi^{(\nu)}$ is contained in the representation with the character $\Xi$ is

$$
\text { (1a) } N_{\nu}=h^{-1} \int \Xi(r) \chi^{(\nu)}(r) d r=h^{-1} \int \chi^{(a)}(r) \chi^{(b)}(r) \cdots \chi^{(\nu)}(r) d r \text {. }
$$

$h$ is the volume of the group if the group is compact; it is the order of the group if this is finite. $r$ is a group element and $\int d r$ indicates the invariant group integration for compact groups, summation over all group elements in the case of finite groups. The extension of the last formula to noncompact groups is an interesting problem on which a great deal of progress has been registered but which, to my knowledge, has not been solved completely.

It is noteworthy that the expression for $N_{\nu}$ is symmetric in its factors. We shall denote it by $(a, b, \cdots, \nu)$. This means, in the case 
of three factors, i.e., the decomposition of the Kronecker product of two representations, that the Kronecker product of $a$ and $b$ contains $c$ just as many times as the Kronecker product of $a$ and $c$ contains $b$, or that of $b$ and $c$ contains $a$.

For three factors, the $N$ are, as a rule, not difficult to calculate. In principle, such a calculation permits obtaining the decomposition of the Kronecker product of any number of factors. This follows from the completeness of the character functions which give the relation

$$
\chi^{(a)}(r) \chi^{(b)}(r)=\sum_{\nu}(a b v) \chi^{(v)}(r) .
$$

We can introduce this into the expression for the decomposition of the threefold product and obtain

$$
\begin{gathered}
(a b c d)=\sum_{\nu}(a b v) \int \chi^{(\nu)}(r) \chi^{(c)}(r) \chi^{(d)}(r) d r \\
=\sum_{\nu}(a b \nu)(\nu c d) .
\end{gathered}
$$

This suggests defining the matrices $M^{*}$

$$
M_{\alpha \beta}^{\kappa}=(\alpha \kappa \beta) .
$$

They are symmetric and their elements are nonnegative integers. Their rows and columns are labeled by the irreducible representations and they all commute, as is evident from the expression for $(a b c d)$. In terms of these matrices, one can write

$$
(a b c \cdots \nu)=\left(M^{b} M^{c} \cdots\right)_{a v}
$$

The order of the $M$ in this expression can be interchanged and there are several other expressions for the left side. None of these expressions shows the total symmetry of the final expression in any obvious way and, in fact, it is not easy to find a general expression (apart from (1a)) for the left side of (5) which is obviously symmetric.

The possibility of decomposing the Kronecker product of two representations, let us say of $D^{(a)}$ and $D^{(b)}$, into definite components does not answer the principal question of interest for the physicist: how is the decomposition done? This amounts to transforming the product of the representation spaces of $D^{(a)}$ and $D^{(b)}$ into the representation spaces of the irreducible components of their Kronecker product. The transformation in question occurs in concrete problems again and again and has been very closely investigated for the group of rotation in three-space. 
The first question which arises is the uniqueness of the transformation. This depends on the uniqueness of the basis vectors in the two spaces to be transformed into each other. Since the basis vectors in both $D^{(a)}$ and $D^{(b)}$ were uniquely specified, this is true also for the direct product of the two spaces. However, if the Kronecker product of $D^{(a)}$ and $D^{(b)}$ contains any representation more than once, not every basis vector in the final space can be specified simply as a definite basis vector of an irreducible $D^{(c)}$. If $D^{(c)}$ occurs in $D^{(a)} \times D^{(b)}(a, b, c)$ times, a unitary transformation of $(a, b, c)$ dimensions will remain free and, if one wants to specify the transformation completely, some added specification of the basis vectors in the final space is necessary. Biedenharn devoted a great deal of thought to this problem, as did also Moshinsky, and the former specified the basis vectors for our problem in the case of the unitary group in $n$ dimensions [19].

In the case of the group of principal interest in physics, the $\mathrm{O}_{3}$ group, the aforementioned difficulty does not arise: all symbols $(a, b, c)$ are either 0 or 1 in this case. Groups of the nature that $(a, b, c)$ can assume only the values 0 or 1 are called, therefore, simply reducible. Both George Mackey and I have devoted a great deal of attention to them [20].

If more than two systems are to be united, i.e., if one is interested in the Kronecker product of more than two representations, the same irreducible representation may occur more than once in the decomposition even in the case of simply reducible groups. The obvious procedure, in order to specify the transformation uniquely, is to proceed step by step, as we did when calculating the value of the multiple symbols $(a b c \cdots \nu)$.

Let us look, first, at the reduction of the Kronecker product of two irreducible representations, $a$ and $b$, of a simply reducible group. The basis vectors of the representation space of $a$ may be denoted by $\alpha_{1}, \alpha_{2}, \ldots$; there are as many of them as is the dimension $l_{a}$ of the representation $a$. An arbitrary one of the $\alpha_{1}, \alpha_{2}, \ldots$ will be denoted by $\alpha$; summation over $\alpha$ then means that each of the $\alpha_{1}, \alpha_{2}, \cdots$ has to be substituted for $\alpha$ and the sum of all the resulting expression taken. A similar remark applies for $\beta$, which is one of the $l_{b}$ basis vectors $\beta_{1}, \beta_{2}, \cdots$ of $b$. In order to express one of the basis vectors $\gamma$ of the representation $c$ (which is contained in the Kronecker product of $a$ and $b)$ in terms of the basis vectors $(\alpha, \beta)$ of the product space of the representation spaces of $a$ and $b$, we have to form a sum

$$
\gamma=\sum_{\alpha \beta} c_{\gamma \alpha \beta}(\alpha, \beta)
$$


Just as into the expression $(a, b, c)$ which we had considered before, the three representations $a, b, c$ enter rather symmetrically into $c_{\gamma \alpha \beta}$. One can express them in the form

$$
c_{\gamma a \beta}=l_{c}^{1 / 2}\left(\begin{array}{lll}
a & b & c \\
\alpha & \beta & \gamma
\end{array}\right)
$$

where $l_{c}$ is the dimension of the representation $c$ and the second factor on the right side is called, for reasons which are unimportant now, a three- $j$-symbol. Except for a possible change in sign, it is invariant with respect to an interchange of the columns. These three- $j$-symbols, or expressions equivalent to them, were also called Clebsch-Gordan coefficients (though the reason for this is mysterious to me), or vector coupling coefficients, which is the name I prefer. They were calculated for $\mathrm{O}_{3}$ in great detail and there are tables for them, similar in thickness to logarithmic tables [21].

Now, if we want to couple three systems together, we can first couple two, and then couple the third to the union of these two. In many cases, this is a physically reasonable procedure because the first two systems may be more strongly coupled to each other than either is to the third. Let us denote the three representations the Kronecker product of which we wish to consider by $a, b$, and $c$. Then, coupling $a$ and $b$, we obtain the expansion of the basis vector $\mu$ of the representation $m$ in the direct product space of their representation spaces

$$
\mu=\sum_{\alpha, \beta} l_{m}^{1 / 2}\left(\begin{array}{ccc}
a & b & m \\
\alpha & \beta & \mu
\end{array}\right)(\alpha, \beta)
$$

We now can take the Kronecker product of the representation $m$ with the remaining representation $c$. The $\delta$ basis vector of the representation $d$ will contain the vector $(\mu, \gamma)$ with the coefficient

$$
l_{d}^{1 / 2}\left(\begin{array}{lll}
m & c & d \\
\mu & \gamma & \delta
\end{array}\right)
$$

so that the vector $\delta$ becomes

$$
\delta=\sum_{\mu \alpha \beta \gamma}\left(l_{\theta} l_{m}\right)^{1 / 2}\left(\begin{array}{lll}
a & b & m \\
\alpha & \beta & \mu
\end{array}\right)\left(\begin{array}{ccc}
m & c & d \\
\mu & \gamma & \delta
\end{array}\right)(\alpha, \beta, \gamma) .
$$

One can again proceed further and couple more and more systems together, i.e., obtain the basis vectors of the irreducible parts of the direct product space of several irreducible representations. 
It is important to note, however, that the vector $\delta$, obtained above, may not be identical with the vector which would have been obtained, had we reduced out, first, the Kronecker product of the representations $a$ and $c$, and coupled one of the irreducible spaces in this Kronecker product with the representation space of $b$. The reason is that a representation $d$ could have been obtained not only by coupling the $m$ part of the direct product spaces of $a$ and $b$ with $c$, but possibly also by coupling another, $m^{\prime}$ part thereof with $c$. Hence, the vector $\delta$ above should be denoted by $\delta^{m}$, its index $m$ specifying the intermediate representation which was then coupled to $c$. Similarly, the vector

$$
\delta^{\prime}=\sum_{\alpha \beta \gamma \mu^{\prime}}\left(l_{d} l_{m^{\prime}}\right)^{1 / 2}\left(\begin{array}{ccc}
a & c & m^{\prime} \\
\alpha & \gamma & \mu^{\prime}
\end{array}\right)\left(\begin{array}{ccc}
m^{\prime} & b & d \\
\mu^{\prime} & \beta & \delta^{\prime}
\end{array}\right)
$$

should be denoted by $\delta^{\prime m^{\prime}}$, its index specifying the intermediate representation which was then coupled to $b$. There is no one-to-one correspondence between the $\delta^{m}$ and the $\delta^{\prime m^{\prime}}$ but each $\delta^{\prime m^{\prime}}$ can be expressed in terms of all the $\delta^{m}$ (and conversely). The coefficients are the same for all basis vectors $\alpha, \beta, \gamma$ and $\delta$ and are equal, apart from the sign and a factor $l_{m}$ to the "six- $j$-symbols", recoupling, or Racah coefficients [22]

$$
\delta^{\prime m^{\prime}}=\sum_{m} l_{m}\left\{\begin{array}{lll}
a & b & m \\
d & c & m^{\prime}
\end{array}\right\} \delta^{m} .
$$

These Racah coefficients also play important roles in a variety of physical problems, atomic and nuclear spectroscopy being only two of them. They have been extensively tabulated and obey a number of symmetry relations: all columns can be interchanged

$$
\left\{\begin{array}{lll}
a & b & e \\
c & d & f
\end{array}\right\}=\left\{\begin{array}{lll}
b & a & e \\
d & c & f
\end{array}\right\}=\left\{\begin{array}{lll}
a & e & b \\
c & f & d
\end{array}\right\} \text { etc. }
$$

Any pair of columns can be reversed

$$
\left\{\begin{array}{lll}
a & b & e \\
c & d & f
\end{array}\right\}=\left\{\begin{array}{lll}
c & d & e \\
a & b & f
\end{array}\right\}=\left\{\begin{array}{lll}
a & d & f \\
c & b & e
\end{array}\right\} \text { etc. }
$$

and there are a number of orthogonality relations which, similar to the preceding symmetry relations, are valid for the Racah coefficients of any simply reducible group. For those of $O_{3}$, Regge has, in addition, proved a further set of relations the deeper cause of which is still somewhat mystifying

$$
\left\{\begin{array}{lll}
a & b & m \\
c & d & m^{\prime}
\end{array}\right\}=\left\{\begin{array}{lll}
\frac{1}{2}(a+c+b-d) & \frac{1}{2}(a-c+b+d) & m \\
\frac{1}{2}(a+c-b+d) & \frac{1}{2}(-a+c+b+d) & m^{\prime}
\end{array}\right\}
$$


if $a, b, c, \cdots$ denote the representations of dimension $2 a+1,2 b+1$, $2 c+1, \cdots$ of $O_{3}$. A similar extension of the relations between the vector coupling coefficients of $\mathrm{O}_{3}$ was also found by Regge [23].

The considerations of the preceding paragraphs have been extended by various authors to the direct product of more than three irreducible representations. Many authors participated in this work (Biedenharn, Edmonds, Ponzano) the motif for which was no longer the facilitation of the solution of problems in physics but the mathematical exploration of a set of intriguing connections [24]. The most complete review of the subject, together with a significant extension of previous results, was given probably by the Lithuanian group of Jucys, Levinsonas and Vanagas [25]. Chakrabarti, Lévy-Nahas and Lévy-Leblonde and the Australian physicist-mathematician-philosopher Kumar, on the other hand, gave expressions for the $\delta$ into which the representations $a, b$, and $c$ of $O_{3}$ entered in a symmetric fashion [26]. Kumar provided such expressions also for irreducible parts of the Kronecker products of more than three irreducible representations of $\mathrm{O}_{3}$.

When concluding this part of the review, one can safely say that the closer scrutiny and more detailed investigation of the direct products of irreducible representations of at least some groups, in particular of $\mathrm{O}_{3}$, led to a number of intriguing relations. They are, perhaps, less general and more concrete than would correspond to modern mathematical taste but, undeniably, many of us physicists enjoyed exploring them.

I do not believe that the subject of the reduction of direct products of representations has been exhausted. There are many other contributions, which were not mentioned [27], and there is a number of questions to which I would like to know the answers. It is true, nevertheless, that the problems which are most important for the physicist have apparently been solved-just as the mathematical problems of crystallography were solved. Instead, a new field for the application of symmetry considerations has turned up and it turned up, appropriately enough, in the most modern part of theoretical physics, in particle theory.

Symmetry problems of particle physics. It is not easy to review the symmetry problems of particle physics because the real problems cannot yet be formulated clearly. The observation which we wish to account for is that there are groups of particles- 8 heavy particles, for instance-which belong to the same representation of the Poincaré group, except that their masses are somewhat different. The aforementioned 8 particles are the best known example; among the 8 
is the proton and the neutron. The other particles are called strangethey have all been discovered in the reasonably recent past.

It seems reasonable to assume that the particles in question are related to each other by some approximate symmetry, and the question is only what that symmetry is. We believe that there is a group which has an 8-dimensional representation and the state vectors of the 8 particles are basis vectors in that representation space. The problem is, physically, what the meaning of the operations of the group is, in the same sense in which the meaning of the operations of the Poincare group is displacement, imparting a velocity, etc. No such meaning is known. It is even doubtful that a real meaning for the operations of the group can be found because the state vectors of the particles in this case do not form a linear space-there is no sense in adding the state vector of a neutron to that of a proton. All this does not, of course, exclude the possibility that the basic equations are mathematically invariant; at least approximately, under a group. It only means that the operations of the group have no direct physical significance. The equations of the oscillator are invariant with respect to an in terchange of the position and velocity coordinates and such invariances have interesting consequences even though the underlying mathematical operation is not meaningful physically.

This is the problem of the physical interpretation of the symmetry. The mathematical problem is to find the group which has an 8-dimensional representation, appropriate for the situation. Gell-Mann and $\mathrm{Ne}^{\prime}$ eman [28] proposed the unimodular unitary group in three dimensions, $S U_{3}$. The first nontrivial real irreducible representation of this is 8-dimensional. What can the group do for us?

If the operations of the group were true symmetry operations, the masses of the 8 particles would be equal. This follows from a theorem of O'Raifertaigh [29] according to which there is no Lie group, containing the Poincaré group as a subgroup, which would have an irreducible representation such that its restriction to the Poincare subgroup would contain a finite number of irreducible representations of this group, with different masses. It then follows that the mass differences must be due to some inaccuracy of the $S U_{3}$ symmetry, some perturbation thereof. The great success of the $S_{3}$ symmetry is then to have suggested a simple perturbation operator the matrix elements of which are in the ratio of the observed mass differences of the 8 particles. I am referring to the Gell-Mann-Okubo mass formula which is valid within about $6 \%$ of the mass differences [30].

The agreement between a mass formula and the observed masses 
would not be in itself convincing. There are several simple perturbation operators and it is not surprising that one led to a conclusion in good agreement with experiment. However, the 8-fold multiplet is not the only one-at least three others could be identified. It seems most unlikely that all this would be coincidence.

I know that my discussion of this subject is too vague to attract the interest of the mathematician. He likes to start with clearly defined premises and arrive at definite conclusions. We surely understand this predilection. However, the task of the physicist is of ten just the opposite: he knows the conclusions, the observed phenomena, and wishes to find the premises from which these follow. A great deal of vagueness is unavoidable in the process and it is, I believe, in spite of its somewhat vague nature, or perhaps just because of it, highly interesting. In addition to those who were already mentioned, Michel in France, Roman in Boston, Gürsey in Turkey, Radicati in Italy, and Pais in New York are some of the most effective contributors thereto [31], but I do not wish to go further into detail. The principal point which the preceding discussion was intended to convey is the fact that, after a long period in which the detailed properties of representations were at the center of interest of the physicist and invariance theorist, attention shifted back to the search for the symmetry group, or symmetry groups, most appropriate for the description of the observed phenomena.

It is a pleasure to thank, in conclusion, Professors V. Bargmann, C. C. Gillispie, and T. S. Kuhn for having called to my attention articles with which I was unfamiliar and for useful discussions.

\section{REFERENCES}

1. J. Willard Gibbs' Elementary principles in statistical mechanics, a finished work 284 pages long, was written in 1901 and published by Yale University in 1902. It was reprinted by Longmans, Green (New York, 1928). An appraisal, by A. Haas (or, rather, two appraisals, a short and a long one) remain worth reading (in Vol. 2 of $A$ commentary on the writings of $J$. Willard Gibbs, Yale University Press, 1936). Gibbs' first writing on the concept to which his name is rightly attached, on phase space, is only one page long. It was published in 1884 (Proc. Amer. Assoc. 33, 57) and is reprinted, as page 16, in Vol. 2 of The scientific papers of Willard Gibbs (Longmans Green, London, 1906).

2. See, e.g., F. P. Bowden and D. Tabor, Friction and lubrication (Wiley, New York, 1956).

3. Il Saggiatore (Volume VI of Le opere di Galileo Galilei, Firenze, 1896) Section 6: Egei e scritto in lingua matematica. ...

4. J. F. C. Hessel's paper was published, originally, in Gehler's Physikalische Wörterbücher (Leipzig, 1830); it is reprinted in Ostwald's Klassiker der exakten Naturwissenschaften No. 89 (Leipzig, 1897), see p. $91 \mathrm{ff}$. 
The history of crystallography was described by P. Groth in his Entwicklungsgeschichte der mineralogischen Wissenschaften (Berlin, 1926). A modern history, very wide ranging, is J. G. Burke's Origins of the science of crystals (University of California Press, 1966).

5. R. J. Haüy's most relevant article is that in the Journ. de Physique 20 (1782), 33. For a complete bibliography, see J. G. Burke, 1. c. p. 190. Actually, Burke expresses doubts (see pp. 83-84) concerning Haüy's independence from his predecessors, in particular from $\mathrm{T}$. Bergman.

6. Actually, N. Steno's De solido intra solidem naturaliter contento dissertationis prodromus (Florence, 1669) only contains the germs of the ideas of the crystal lattice.

7. A. Schönflies, Kristallsysteme und Kristallstruktur (Leipzig, 1891); E. S. Fedorov, Zap. Min. Obsh. (Trans. Min. Soc.) 28 (1891), 1.

8. P. Groth, Physikalische Krystallographie (W. Engelman, Leipzig, 1905). A more openly group theoretical attitude is adopted by W. Voigt in his Lehrbuch der Kristallphysik (B. G. Teubner, Leipzig, 1910).

9. According to classical, that is nonrelativistic theory, the equations of motion remain invariant if the three positional coordinates $x_{i}(i=1,2,3)$ are subjected to a Galilei transformation

$$
x_{i}^{\prime}=\sum_{k=1}^{8} O_{i k} x_{k}+v_{i} t+a_{i} \quad(i=1,2,3)
$$

where the $O_{i k}$ form a $3 \times 3$ orthogonal matrix and the time $t$ is invariant or subject to a change of origin

$$
t^{\prime}=t+a_{0} .
$$

The vector $v$ gives the velocity of the two coordinate systems with respect to each other; the vector $a$ the displacement of the origin of the second coordinate system with respect to the first one at $t=0$. The corresponding invariance transformation of relativity theory, the Poincare transformation, treats the time more on a par with the space coordinates. It introduces, instead of $t$, the variable $x_{0}=c t$ (where $c$ is the velocity of light). The transformation is, in terms of these

$$
x_{i}^{\prime}=\sum_{k=0}^{3} L_{i k} x_{k}+a_{i} \quad(i=0,1,2,3)
$$

where $L$ is a Lorentz transformation, i.e. an element of $O(1,3)$. The Poincaré transformation is called also inhomogeneous Lorentz transformation.

10. This is now a commonplace statement. It is due, originally, in the precise form stated, to J. v. Neumann. See his Mathematische Grundlagen der Quantenmechanik (J. Springer, Berlin, 1932). English translation by R. T. Beyer, Princeton University Press, 1955.

11. This phase space is the mathematical structure introduced by W. Gibbs and mentioned in Reference [1].

12. V. Bargmann, Ann. of Math. 59 (1954), 1.

13. This was shown, first, by the present writer, Ann. of Math. 40 (1939), 149.

14. Gelfand, Naimark, and their collaborators are extremely prolific writers. The most important books, from our point of view, are: I. M. Gelfand, R. A. Minlos, and Z. Y. Shapiro, Representations of the rotation and Lorentz groups and their applications, Macmillan, New York, 1963; M. A. Naimark, Linear representations of the Lorentz group, Pergamon Press, London, 1964; I. M. Gelfand and M. A. Neumark, Unitäre Darstellungen der klassischen Gruppen, Akademie Verlag, Berlin. See also the articles 
of these authors in Vols. 2 and 36 of the Amer. Math. Soc. Translations (Amer. Math. Soc., Providence, R. I., 1956 and 1964).

Harish-Chandra's articles are too numerous for a complete listing and no review of his results is available in book form. His early papers appeared in the Proc. Nat. Acad. Sci. (Vols. 37-40, 1951-54). Among his later writings, we wish to mention his Invariant eigendistributions on a semisimple Lie algebra (Inst. Hautes Etudes Sci. Publ. Math. No. 27, p. 5) and his article in Ann. of Math. 83 (1966), 74.

15. A. Young, Proc. London Math. Soc. 33 (1900), 97; 34 (1902), 361; G. Frobenius, Sitzungsberichte Preuss. Akad. Wiss. 1903, p. 328; I. Schur, ibid. 1908, p. 64. See for a physicist's approach, A. J. Coleman's article in Advances of quantum chemistry (Academic Press, New York, 1966).

16. To be published in the Racah Memorial Volume (North-Holland, Amsterdam, 1968), p. 131.

17. R. de L. Kronig, Zeits. f. Physik 31 (1925), 885; 33 (1925), 261; H. N. Russell, Proc. Nat. Acad. Sci. 11 (1925), 314; A. Sommerfeld and H. Hönl, Sitzungsberichte Preuss. Akad. Wiss., 1925, p. 141.

18. The Kronecker product as the representative of the union of two physical systems was implicit already in Schrödinger's papers. See his Abhandlungen zur Wellenmechanik, Leipzig, 1927. It was made explicit and precise by von Neumann, [10, Chapter VI, \$2].

19. L. C. Biedenharn, J. Math. Phys. 4 (1963), 436 and subsequent papers, ending with L. C. Biedenharn, A. Giovanni. J. D. Louck, ibid. 8 (1967), 691. M. Moshinsky, ibid. 7 (1966), 691, J. G. Nagel and M. Moshinsky, ibid 6 (1965), 682 and M. Moshinsky ibid. 7 (1966), 691, M. Kushner and J. Quintanilla, Rev. Mex. de Fisica 16 (1967), 251. For a summary, see L. C. Biedenharn, Racah Memorial Volume [16, p. 173].

20. E. P. Wigner, Amer. J. Math. 63 (1941), 57. This and a more detailed paper are reprinted in Quantum theory of angular momentum, L. C. Biedenharn and H. Van Dam, Editors, Academic Press, New York, 1965. This volume contains reprints of several articles which played an important role in the formation of the ideas of physicists on representation theory, in particular also articles of G. Racah. G. W. Mackey, Amer. J. Math. 75 (1953), 387; Pacific J. Math. 8 (1958), 503. See also his Mathematical foundations of quantum mechanics Benjamin, New York, 1963.

21. As far as this writer was able to ascertain, the first calculation of these coefficients is given in his Gruppentheorie und ihre Anwendungen etc. (Friedr. Vieweg, Braunschweig, 1931) Chapter XVII. See also the English translation by J. J. Griffin (Academic Press, New York, 1959). The more symmetric expressions for these quantities, the three-j-symbols, were introduced in the article which is reprinted in the Quantum theory of angular momentum [20, pp. 89-133]. For an extensive tabulation of the numerical values of these coefficients, see, e.g., Rotenberg, Bivins, Metropolis, Wooten, The 3-j and 6-j symbols, Technology Press, MIT, Cambridge, 1959.

22. The first publications containing recoupling (now called Racah) coefficients are due to G. Racah. His work was clearly independent of earlier considerations of the present writer (cf. the second article of [20]). They arose in connection with problems of atomic spectra. Cf. Phys. Rev. 61 (1942), 186; 62 (1942), 438; 63 (1943), $367 ; 76$ (1949), 1352. These articles are all reprinted in the Quantum theory of angular momentum [20]. For a numerical table of these coefficients, also called $6-j$-symbols, see $[21]$.

23. T. Regge, Nuovo Cimento 11 (1959), 116; 10 (1958), 544. An extension of these relations was given by $R$. T. Sharp.

24. L. C. Biedenharn, J. M. Blatt, M. E. Rose, Revs. Mod. Phys. 24 (1952), 249; 
A. R. Edmonds, Angular momentum in quantum mechanics, Princeton University Press, 1957; G. Ponzano, Nuovo Cimento 35 (1965), 1231 ; 36 (1965), 385; G. Ponzano and T. Regge, Racah Memorial Volume [16].

25. A. Jucys, J. Levinsonas, V. Vanagas, Mathematical apparatus of the theory of angular momentum. English translation published by the Israel Program of Scientific Translations, Jerusalem, 1962. The original, in Russian, was published in Vilnius (Vilna) in 1960. See also W. T. Sharp's Racah algebra and the contraction of groups, Atomic Energy of Canada Ltd. report 1098 (1960).

26. A. Chakrabarti, Ann. Inst. Henri Poincaré 1 (1964), 301; K. Kumar, Austral. J. Phys. 19 (1966), 719; J. M. Lévy-Leblond and M. Lévy-Nahas, J. Math. Phys. 6 (1965), 1372.

27. A full listing of all the contributions to the subject would require several pages. Furthermore, some noncompact Lie groups, in particular also the Poincaré group, were the subjects of investigations similar to those described for $\mathrm{O}_{3}$. See, for instance, J. Ginibre, J. Math. Phys. 4 (1963), 720; J. R. Derome and W. T. Sharp, ibid. 6 (1965), 1584; D. R. Tompkins, ibid. 8 (1967), 1502.

Articles dealing with other properties of the representations of the Poincare group and also extensions thereof include J. L. Lomont and M. E. Moses, J. Math. Phys. 5 (1964), 294; 8 (1966), 837; I. Raszillier, Nuovo Cimento 38 (1965), 1928; J. M. Lévy-Leblond, ibid. 40 (1965), 748; C. George and M. Lévy-Nahas, J. Math. Phys. 7 (1966), 980; J. C. Guillot and J. L. Petit, Helv. Phys. Acta 39 (1966), 281; V. Berzi and V. Goroni, Nuovo Cimento 57 (1967), 207; S. Ström, Ark. Fys. 34 (1967), 215; J. Nilsson and A. Beskow, ibid. 34 (1967), 307; H. Joos and R. Schrader, Comm. Math. Phys. 7 (1968), 21 (the characters of the representations); A. Kihlberg, Nuovo Cimento 53 (1968), 592.

Other articles investigate more complex noncompact Lie groups as they underlie the de Sitter space (the groups $O(4,1)$ and $O(3,2)$, or the situation at very high energy $O(4,2)$ or $U(2,2))$. See the very early paper of L. H. Thomas, Ann. of Math. 42 (1941), 113. Corrections to this paper and extensions thereof were given by T. D. Newton, Ann. of Math. 51 (1950), 730 and by J. Dixmier, Bull. Soc. Math. France 89 (1960), 9. Other contributions are due to J. B. Ehrman, Proc. Cambridge Philos. Soc. 53 (1957), 290; several articles of A. Kihlberg and S. Ström, including some mentioned before but in particular Ark. Fys. 31 (1966), 491; W. Rühl, Nuovo Cimento 44 (1966), 572; A. Chakrabarti, J. Math. Phys. 7 (1966), 949; A. J. Macfarlane, L. O'Raifeartaigh and P. S. Rao, ibid. 8 (1967), 536; O. Nachtman, Acta Physica Austriaca 25 (1967), 118; H. Bacry, Comm. Math. Phys. 5 (1967), 97. The Trieste Institute of the International Atomic Energy Agency is intensely working on the subject and has issued many reports thereon. The authors include R. Delbourgo, A. Salam and J. Strathdee; R. L. Anderson, R. Raczka, M. A. Rashid and P. Winternitz; D. T. Stoyanov and I. T. Todorov.

A rather complete review of the more important articles from the mathematical point of view, up to 1965, was presented by G. A. Pozzi, Nuovo Cimento Suppl. 4 (1966), 37. See also H. Baumgärtel, Wiss. Z. Humboldt Univ., Berlin, Math.-Naturw. Reihe 13 (1964), 881.

28. Y. Ne'eman, Nuclear Physics 26 (1961), 222; M. Gell-Mann, Phys. Rev. 125 (1962), 1067. See also their reprint collection and review, The eightfold way, Benjamin, New York, 1964.

29. L. O'Raifeartaigh, Phys. Rev. 139B (1965), 1952. A mathematically more precise formulation of the O'Raifeartaigh theorem was given by R. Jost, Helv. Phys. 
Acta 39 (1966), 369. For an extension of the theorem, see I. Segal, J. Functional Anal. 1 (1967), 1; A. Galindo, J. Math. Phys. 8 (1967), 768.

30. S. Okubo, Progr. Theor. Phys. 27 (1962), 949; M. Gell-Mann, Phys. Rev. 125 (1962), 1067.

31. The interested reader will find an illuminating introduction to, and a collection of, articles dealing with the problems of the extension of the Poincare group in F. J. Dyson's Symmetry groups in nuclear and particle physics, Benjamin, New York, 1966. A more mathematically oriented review of the "higher symmetries" is given in M. Gourdin's book, Unitary symmetries and their applications to high energy physics, North-Holland, Amsterdam, 1967. See also A. O. Barut, Proc. Seminar on High Energy Physics and Elementary Particles, Trieste, 1965 (International Atomic Energy Agency, Vienna, 1965).

Princeton UnIVERsity 